\title{
Research on Priority Evaluation Model of Project Associated Clusters
}

\author{
Yuxi Zhang 1,*, Yingjing He ${ }^{2}$, Keping Zhu ${ }^{2}$, Wei Fan ${ }^{3}$, Zhongfu Tan ${ }^{3}$ \\ ${ }^{1}$ China Electric Power Research Institute, Beijing, 100192, China \\ ${ }^{2}$ State Grid Zhejiang Electric Power Company Limited Economic \& Technical Research Institute, Hangzhou, 310001, China \\ ${ }^{3}$ North China Electric Power University, Beijing, 102206, China
}

\begin{abstract}
At present, there are still some urgent problems to be solved, such as repeated reserve projects, unreasonable timing arrangement of investment in project execution, poor effect of capital use and poor process connection. In this paper, through the related cluster demonstration of the comprehensive plan projects, we strengthen the pre-management and control of the reserve projects from the source, and improve the quality and efficiency of the comprehensive plan management. Firstly, this paper classifies the projects based on the perspective of business association, establishes the association cluster, and then constructs the priority evaluation model of the project association cluster with the help of the portfolio management theory, so as to maximize the project benefit.
\end{abstract}

\section{Introduction}

In recent years, the State Council has issued a series of documents aimed at streamlining administration and delegating power, improving the efficiency and fairness of resource allocation and creating a sound business environment. At the same time, the adjustment of new regulations on investment supervision of central enterprises and the promotion of streamlining administration and delegating power also highlight the importance of all-round and whole-process supervision, which puts forward higher requirements for enterprises to further improve their comprehensive plan management and continuously improve management efficiency and benefits.

Literature [1], fuzzy clustering algorithm is used to achieve clustering analysis of similar alarms. Literature [2] proposes a fuzzy grey correlation analysis and evaluation method for cluster project bid section division. Literature [3] summarized the deficiencies of the current mainstream recommendation algorithms, and proposed a new collaborative filtering recommendation algorithm to address the defects. Literature [4] points out that the objectives of portfolio management mainly include the number of project portfolios, the value of project portfolios, the optimal allocation of resources among multiple projects, the sharing of information and resources among multiple projects, and the improvement of the overall value of the organization. Literature [5] points out the constraints of portfolio management, which mainly include the organization of portfolio management, resources and technologies that can be shared among multiple projects, effective decomposition and commitment of the responsibility system at the level of functional departments and projects, rapid information transmission, effective sharing and mining analysis among multiple projects.

Based on the above analysis, the goal of project association analysis is to dig out the interrelationship hidden in project data, analyze the given project and record set through association rules, get the correlation between projects, and put forward reference opinions on the division and collection of different projects of the company.

\section{Association type analysis of reserve items}

Based on the business perspective, this paper analyzes the project association mode, from three aspects of electrical association, facility association and function association.

Electrical connection. Electrical correlation is based on the principle of electrical connection. Projects with close electrical connection are correlated with each other and are progressively linked to $35 \mathrm{KV}$ projects in the order from high voltage grade to low voltage grade.

Facility and equipment association. Facilities and equipment association refers to the infrastructure such as roads, railways, ports, office buildings or production houses as the associated subject, and the new construction, renovation or overhaul that will take place on the same associated subject is associated with the purchase of office or production equipment not included in the project.

Functional correlation. According to the national policy and the company's key work requirements, the

\footnotetext{
* Corresponding author: $18811612979 @ 163 . c o m$
} 
projects with the same construction and investment objectives are related to each other.

Based on different entry points, reserve projects will have different ways of association, and the project association cluster will be established according to the way of project association, which lays a good foundation for the demonstration of project cluster.

\section{Multidimensional project priority evaluation model}

The purpose of the multi-dimensional project association demonstration is to optimize the cluster combination management of the project, and realize the project timing optimization, the highest efficiency and the maximum benefit. The project priority evaluation is helpful for sorting out the correlation of all projects and establishing the project portfolio model ${ }^{[6]}$.

\subsection{Establishment of evaluation system}

The evaluation index system for portfolio optimization is an evaluation system formed by identifying some common evaluation factors among various projects. The evaluation system established in this paper is shown in Figure 1.

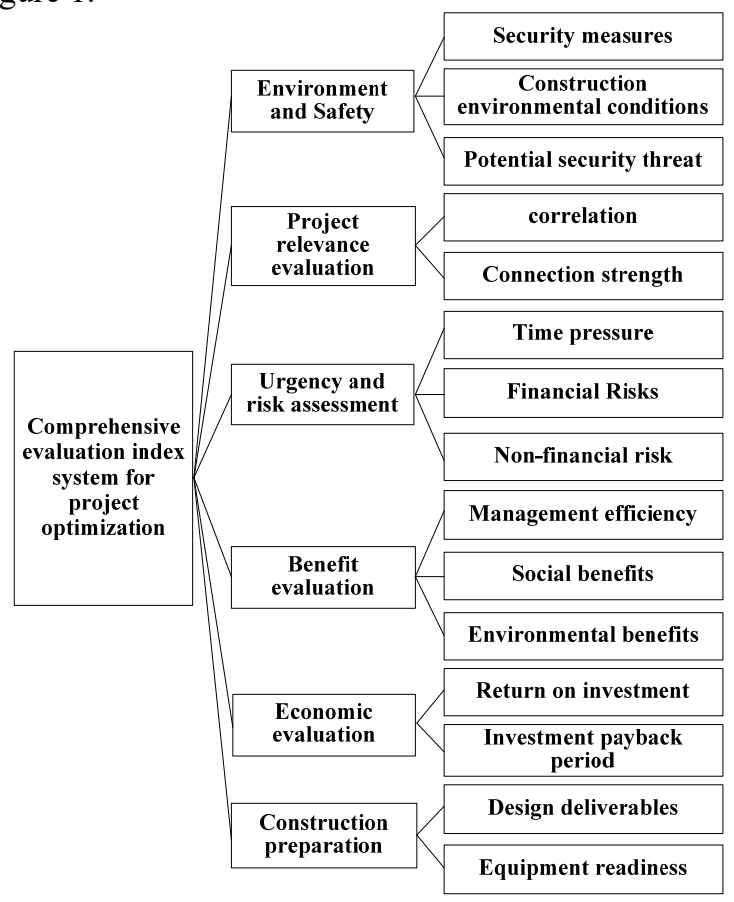

Figure. 1 Multi-dimensional project optimization evaluation index system

\subsection{Multi-dimensional portfolio optimization model}

In order to improve the accuracy and scientificity of the comprehensive evaluation, this paper adopts the grey hierarchy evaluation method to integrate the scattered information of each expert into a weight vector describing different degrees of the grey category, and then carries on the single value processing on the basis of this, obtains the comprehensive evaluation value, and then can prioritize the project.

According to the principle of Analytic Hierarchy Process (AHP), the three-level evaluation index system is formed by the arrangement of the highest level (target $W$ ), the middle level (first-level evaluation index $U_{i} ; i=1,2, \cdots, m$ ) and the lowest level (second-level evaluation index $\left.V_{i j} ; i=1,2, \cdots, m, j=1,2, \cdots, n\right)$.The serial number of the evaluation project is $S(S=1,2, \cdots, q)$, $W^{(s)}$ which represents the optimal evaluation value of the $S$ project; $U$ represents the set composed of first-level evaluation index $U_{i}$; , denoted as $U=\left\{U_{1}, U_{2}, \cdots, U_{m}\right\}$.

$V_{i}(i=1,2, \cdots, m)$ represents the set composed of secondlevel evaluation index $V_{i j}$, denoted as $V_{i}=\left\{V_{i 1}, V_{i 2}, \cdots, V_{m j}\right\}$.Then the specific steps of the hierarchical grey evaluation method are as follows:

1) According to the index system, determine the evaluation standard

The evaluation index $V_{i j}$ was divided into 4 levels and assigned with points of $4,3,2$ and 1 . When the index level was between adjacent levels, the evaluation index $\mathrm{V}$ was assigned with points of 3.5, 2.5 and 1.5, The order from high to low indicates that the index rating is from good to bad.

2) Determine the weight of evaluation index $U_{i}$ and $V_{i j}$

The idea of analytic hierarchy process is used to determine the weight of evaluation indexes $U_{i}$ and $V_{i j}$ by establishing judgment matrix and solving eigenvalue of matrix. The weight reflects the importance of evaluation indexes $U_{i}$ and $V_{i j}$ to target $W$. The greater the weight, the higher the importance.

3) Organize evaluation expert scores

It is assumed that there are $p$ evaluation experts with serial number $k, k=1,2, \cdots, p$, and the evaluation index $V_{i j}$ of the s-th project is scored by the $p$ experts respectively according to the grading standard.

4) Find the evaluation sample matrix

Assume that $d_{i j k}^{(s)}$ is the score given by the kth expert to the evaluation index $V_{i j}$ of the Sth project, then the evaluation sample matrix $D^{(s)}$ of the Sth project is

$$
D^{(s)}=\left[\begin{array}{ccccc}
d_{111}^{(s)} & d_{111}^{(s)} & \cdots & d_{111}^{(s)} & V_{11} \\
\vdots & \vdots & \ddots & \vdots & \vdots \\
d_{1 n_{1} 1}^{(s)} & d_{1 n_{2} 2}^{(s)} & \cdots & d_{1 n_{1} p}^{(s)} & V_{1 n_{1}} \\
d_{211}^{(s)} & d_{212}^{(s)} & \cdots & d_{21 p}^{(s)} & V_{21} \\
\vdots & \vdots & \ddots & \vdots & \vdots \\
d_{2 n_{1} 1}^{(s)} & d_{2 n_{2} 2}^{(s)} & \cdots & d_{2 n_{2} p}^{(s)} & V_{2 n_{2}} \\
\vdots & \vdots & \ddots & \vdots & \vdots \\
d_{m 11}^{(s)} & d_{m 12}^{(s)} & \cdots & d_{m 1 p}^{(s)} & V_{m 1} \\
\vdots & \vdots & \ddots & \vdots & \vdots \\
d_{m n_{m} 1}^{(s)} & d_{m n_{m} 2}^{(s)} & \cdots & d_{m n_{m} p}^{(s)} V_{m n_{m}}
\end{array}\right]=\left(d_{i j k}^{(s)}\right) p \sum n_{i}
$$




$$
i=1,2, \cdots, m ; j=1,2, \cdots, n_{m} ; k=1,2, \cdots, p
$$

$5)$ Determine the evaluation grey category

Set the gray class serial number as $e(e=1,2,3,4)$, and use the whitening weight function to calculate the gray evaluation matrix:

When $e=1$, the grey category is evaluated as "excellent", that is $\otimes_{1} \in(4, \infty)$, its expression is:

$$
f_{1}\left(d_{i j k}^{(s)}\right)=\left\{\begin{array}{c}
\frac{d_{i j k}^{(s)}}{4}, d_{i j k}^{(s)} \in(0,4) \\
1, d_{i j k}^{(s)} \in(4, \infty) \\
0, d_{i j k}^{(s)} \in(0, \infty)
\end{array}\right.
$$

When $e=2$, the grey category is evaluated as "good", that is $\otimes_{2} \in(0,3,6)$, its expression is:

$$
f_{2}\left(d_{i j k}^{(s)}\right)=\left\{\begin{array}{c}
\frac{d_{i j k}^{(s)}}{3}, d_{i j k}^{(s)} \in(0,3) \\
\frac{d_{i j k}^{(s)}-6}{-3}, d_{i j k}^{(s)} \in(3,6) \\
0, d_{i j k}^{(s)} \in(0,6)
\end{array}\right.
$$

When $e=3$, the grey category is evaluated as "general", that is $\otimes_{3} \in(0,2,4)$, its expression is:

$$
f_{3}\left(d_{i j k}^{(s)}\right)=\left\{\begin{array}{cl}
\frac{d_{i j k}^{(s)}}{2}, & d_{i j k}^{(s)} \in(0,2) \\
\frac{d_{i j k}^{(s)}-4}{-2}, & d_{i j k}^{(s)} \in(2,4) \\
0, d_{i j k}^{(s)} \in(0,4)
\end{array}\right.
$$

When $e=4$, the grey category is evaluated as "poor", that is $\otimes_{4} \in(0,1,2)$, its expression is:

$$
f_{4}\left(d_{i j k}^{(s)}\right)=\left\{\begin{array}{c}
1, d_{i j k}^{(s)} \in(0,1) \\
\frac{d_{i j k}^{(s)}-2}{-1}, d_{i j k}^{(s)} \in(1,2) \\
0, d_{i j k}^{(s)} \bar{\in}(0,2)
\end{array}\right.
$$

6) Grey evaluation coefficient calculation

Grey evaluation coefficient $x_{i j k}^{(s)}$ refers to the grey evaluation coefficient of the S-th project belonging to the e-th evaluation grey for the evaluation index $V_{i j} x_{i j k}^{(s)}=\sum_{k=1}^{p} f_{e}\left(d_{i j k}^{(s)}\right)$. Total gray evaluation coefficient $x_{i j}^{(s)}$ refers to the total gray evaluation coefficient of the S-th project for the evaluation index ${ }^{V_{i j}}, x_{i j}^{(s)}=\sum_{e=1}^{4} x_{i j e}^{(s)}$.

7) Grey evaluation weight vector and weight matrix calculation
For the S-th project, the evaluation index $V_{i j}$ belongs to the grey evaluation weight $r_{i j e}^{(s)}=\frac{x_{i j e}^{(s)}}{x_{i j}^{(s)}}$ of the e-th grey category. The grey evaluation weight vector $r_{i j}^{(s)}: r_{i j}^{(s)}=\left(r_{i j 1}^{(s)}, r_{i j 2}^{(s)}, r_{i j 3}^{(s)}, r_{i j 4}^{(s)}\right)$ of $V_{i j}$ of item S-th. The grey evaluation weight matrix $R_{i}^{(s)}=\left(r_{i 1}^{(s)}, r_{i 2}^{(s)}, \cdots, r_{i n_{i}}^{(s)}\right)$ of the index $V_{i j}$ to which $V_{i}$ of the S-th project belongs.

8) Make a comprehensive evaluation of $V_{i}$

The comprehensive evaluation result $B_{i}^{(S)}, B_{i}^{(S)}=A_{i} R_{i}^{(S)}$ is obtained by making comprehensive evaluation on $V_{i}$ of the S-th evaluation item.

9) Make a comprehensive evaluation of $U$

According to the comprehensive evaluation result $B_{i}^{(S)}$ of $V_{i}$, the index $U_{i}$ to which $U$ belongs to the S-th project is obtained. For the grey evaluation weight matrix $R^{(s)}=\left(B_{1}^{(s)}, B_{2}^{(s)}, \cdots, B_{m}^{(s)}\right) \quad$, the comprehensive evaluation result of the index $U$ of the S-th project is.

10) Comprehensive evaluation value calculation

When $\mathrm{e}=1$, take 4 ; when $\mathrm{e}=2$, take 3 ; when $\mathrm{e}=3$, take 2 ; and when $\mathrm{e}=4$, take 1 . Then, the value vector $C=(4,3,2,1)$ of each evaluation grey class grade is evaluated. $W^{(s)}=B^{(s)} C^{T}$ is the evaluation value of the comprehensive evaluation of the S-th project.

11) Determine project cluster priorities

The cluster's comprehensive evaluation score is the average of all the projects in the cluster. The cluster is ranked from high to low according to the comprehensive evaluation score, that is, the priority of the project cluster is established.

\section{Example analysis}

\subsection{Basic data}

Taking 3 projects and 6 evaluation experts as examples, multi-level grey evaluation model is used to optimize and evaluate the projects in the project portfolio. The weights of the first level evaluation index $u$ and the second level evaluation index $\mathrm{v}$ were determined by analytic hierarchy process. Firstly, the importance comparison scale of the judgment matrix is established, as shown in the following table 1.

Experts judge the importance degree of six first-level indicators, including environment and safety, project relevance evaluation, urgency and risk evaluation, benefit evaluation, economic evaluation, and construction preparation.

According to the consistency calculation, $\mathrm{CR}$ is 0.0332 less than 0.1 , passing the consistency test.Then repeat this process, calculate the weight of the secondlevel index relative to the first-level index, and check the consistency. Finally, combine the weight of the firstlevel index relative to the target layer and the weight of the second-level index relative to the criterion layer to get the comprehensive weight of each index, as shown in the figure 2 . 
Table 1 Importance comparison scale table

\begin{tabular}{|c|c|}
\hline Scale & Define \\
\hline 1 & The $\mathrm{i}$ factor is as important as the $\mathrm{j}$ factor \\
\hline 3 & Factors $\mathrm{i}$ and $\mathrm{j}$ are slightly more important \\
\hline 5 & Factors $\mathrm{i}$ and $\mathrm{j}$ are more important \\
\hline 7 & The i factor and the $\mathrm{j}$ factor are very important \\
\hline 9 & $\begin{array}{c}\text { The i factor and the } \mathrm{j} \text { factor are absolutely } \\
\text { important }\end{array}$ \\
\hline $\begin{array}{c}\text { The } \\
\text { bottom }\end{array}$ & $\begin{array}{c}\text { The scale value corresponding to the } \\
\text { intermediate state between the above two } \\
\text { judgments }\end{array}$ \\
\hline \begin{tabular}{c} 
Factor $\mathrm{j}$ is more important than factor $\mathrm{i}$ \\
\hline
\end{tabular}
\end{tabular}

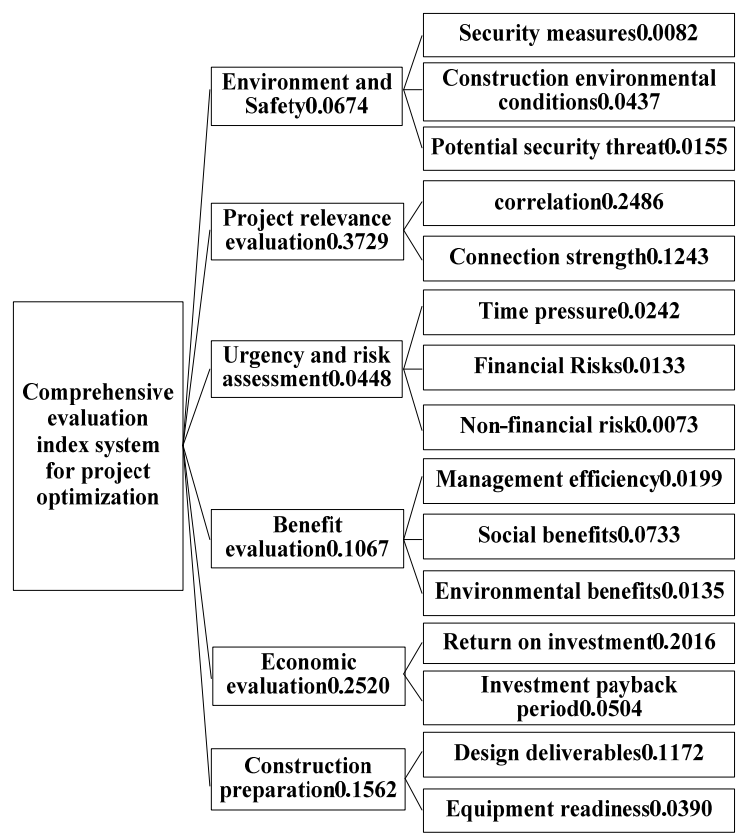

Figure. 2 The comprehensive weight of each indicator

These 3 items were scored by 6 evaluation experts according to the grading standard. Sample according to the expert grading, the evaluation matrix, and then calculate the grey evaluation coefficient, and to get a evaluation of grey evaluation weight matrix of a class, the final comprehensive evaluation of project priorities of project 1 to $W^{(1)}=0.716127$, the integrated evaluation project comprehensive evaluation value of 2 to $W^{(2)}=0.789621$, item 3 of the comprehensive evaluation value of $W^{(3)}=0.802693$, and the comprehensive evaluation value.

The average score of $W^{(1)}, W^{(2)}$ and $W^{(3)}$ is calculated as the comprehensive evaluation score of cluster 1 . Because all evaluation experts agree that each $V_{i j}$ index score of the project is 2 points (qualified score), corresponding to $\mathrm{W}$ of 0.6122 , the cluster can be considered qualified and the project cluster can be implemented when the optimal evaluation value of the cluster is greater than 0.6122.In the same way, the comprehensive evaluation scores of Cluster 2 and Cluster 3 are obtained. The comprehensive evaluation scores are arranged from the highest to the lowest. The higher the scores are, the higher the priority of the project is, that is, the priority of the project cluster is established.

\section{5 conclusion}

In this paper, a model of project portfolio optimization is established to make the structure of comprehensive planning project scientific and reasonable. An example of project optimization evaluation and analysis process is given through numerical calculation. Combined with the development of comprehensive planning projects in power companies, the priority evaluation model of associated cluster can effectively promote the continuous improvement of comprehensive planning management quality.

\section{Acknowledgments}

The work is supported by the State Grid Corporation Project "Research on the project association demonstration system based on multi-element business feature mining" (Funding Contract Number : 1400202055132A-0-0-00)

\section{References}

1. Yang, F. Study on clustering fusion and association analysis of alarm information in heterogeneous networks [D]. Xidian University, 2015.

2. Zheng, X.Z., Guo, Y. W.,Zhu, Z. R. Bid division of cluster project based on fuzzy grey correlation analysis [J]. South-North Water Diversion and Water Resources Science and Technology, 2016, 14(02): 172-176+81.

3. Lin, P., Collaborative Filtering Recommendation Algorithm Based on Grey Relational Clustering [D]. Nanjing University of Posts and Telecommunications, 2015.

4. Robert G. Cooper, Scott J. Edgett. Portfolio Management for New Products: Picking the Winners [J]. Working paper, 2001, (10): 19-25.

5. Suvi Elonen, Karlos Artto. Problems in Managing Internal Development Projects in Multi-Project Environments [J]. International Journal of Project Management, 2003, 21(6): 395-402.

6. Dong, J. X., Wang, J. W.,Gong, Q. M., et al. Research on the priority evaluation model of the associated cluster of reserve items [J]. Modern Management, 2019, 9(1): 40-50. 